\title{
Recht auf Internet
}

\author{
Kai v. Lewinski"
}

A. Internet als Gegenstand des Rechts.... 71

B. TK-Infrastruktur als Voraussetzung des Internets...

I. Gewährleistung einer TK-Infrastruktur

II. Gewährleistung eines TK-Zugangs. . 74

1. Zugangsmöglichkeit............. 74

2. Leistungsrechtlicher Anspruch auf Internetzugang .............. 74

III. Abwehrrechtlicher Schutz........... 77

1. Nur randständiger Schutz durch technikbezogene Grundrechte... 77

2. Allgemeine Handlungsfreiheit... 77

IV. Zwischenergebnis .................. 78

C. Verbreitung von und Zugang zu Inhalten im Internet...................... 78

I. Gewährleistungsgehalte............ 78

1. Gewährleistung der demokratischen Willensbildung...

2. Gewährleistung der Persönlichkeitsentfaltung und Wirtschaftstätigkeit . . .

3. Sozialstaatliche Gewährleistung gesellschaftlicher Teilhabe.......

4. Keine Gewährleistung des eGovernment

5. Keine Gewährleistung einer eCommerce-Infrastruktur ........

II. Leistungsansprüche ..................

III. Abwehrrechtlicher Schutz .......... 83

1. Zerklüftetheit der Schutzbereiche.... a. Meinungsfreiheit........... 84

b. Medienfreiheit.............. 85

c. Kunst- und Wissenschaftsfreiheit....

d. Nachfrager- und Empfängerschutz teilweise nur nach Art. 2 Abs. 1 GG........... 86

e. Kein Meinungsschutz für Internetdienste ....

f. Unvollkommener Schutzbereich in Bezug auf das Internet...................... 87

2. Eingriff..................... 88

3. Schranke..................... 88

4. Zensurverbot ................. 89

5. Internetsperre als Grundrechtsverwirkung?................... 89

6. Strukturelle Schwächen des grundrechtlichen Schutzes des Internets..................... 90

D. Struktur eines Rechts auf Internet.... 90

I. Unterschied zwischen technischem und inhaltlichem „Recht auf Internet“............................ 91

II. Grundsatz des freien Informationsflusses............................ 91

E. Normative Umsetzung eines Rechts auf Internet .......................... 91

F. Fazit ............................ 94

Ist ein „Recht auf Internet“ nur ein politisches Schlagwort? ${ }^{1}$ Einen eindeutigen rechtlichen Gehalt hat es jedenfalls nicht. ${ }^{2}$ Das Grundgesetz enthält keine ausdrücklichen

* PD Dr. Kai v. Lewinski ist Privatdozent für Staats- und Verwaltungsrecht, Finanzrecht, Rechtsgeschichte der Neuzeit an der Juristischen Fakultät der Humboldt-Universität zu Berlin. Bei dem Text handelt es sich um die aktualisierte und um Nachweise ergänzte Fassung des Habilitationsvortrags des Verfassers. - Besonderer Dank gilt Herrn Ass. jur. Holger Greve für die reichen Anregungen zu aktuellen Entwicklungen.

1 So halten nach einer Umfrage der BBC 80 \% der Befragten den Zugang zum Internet für ein Grundrecht (s. F.A.Z. v. 23.7.2010, S. 31); s. auch die 3. These („Recht auf freie Entfaltung im Internet“) und die 7. These („Basisinfrastruktur unseres Zusammenlebens“) der 14 Thesen zu den Grundlagen der gemeinsamen Netzpolitik der Zukunft, die Bundesinnenminister Th. de Maizière am 22.6.2010 vorgelegt hat (abgedr. in RDV 2010, S. 187 ff.). - Zu entsprechenden Forderungen eines „Rechts auf Internet“ in Deutschland M. Kloepfer, Verfassungsrecht, Bd. 1, München 2011, $\mathbb{2}$ Rn. 228.

2 Dazu jetzt A. Roßnagel (Hrsg.), Elektronische Medien zwischen Exklusivität und Grundversorgung, Baden-Baden 2010; früh schon W. Mecklenburg, Internetfreiheit, ZUM 1997, S. 525 ff. 
Bestimmungen, das Völkerrecht macht keine Vorgaben, ${ }^{3}$ und gerichtliche Entscheidungen fallen uneinheitlich aus. So steht die sozialgerichtliche Rechtsprechung bislang auf dem Standpunkt, dass das Internet nicht zum Existenzminimum gehört. ${ }^{4}$ Arbeitsgerichtlich wird ein Anspruch des Betriebsrats auf Internetzugang dagegen heute bejaht. ${ }^{5}$ Das Bundesverfassungsgericht hat zu einem „Grundrecht auf Internet“ noch nicht unmittelbar Stellung genommen. Allerdings ist bis zu ihm gedrungen, dass die „Nutzung des Internet durch große Kreise der Bevölkerung mehr und mehr [der] Normalfall" ist. ${ }^{6}$

Tatsächlich muss die Antwort differenziert ausfallen. Und dies nicht nur, weil wir uns im Übergang zur Informationsgesellschaft befinden, sondern weil das Internet ein mehrschichtiges Phänomen ist, und weil ein „Recht auf Internet“ Gewährleistungspflichten, Leistungs- und Abwehrgehalte sowie objektive Wertentscheidungen umfasst.

\section{A. Internet als Gegenstand des Rechts}

Bevor aber die Frage untersucht wird, ob es ein Recht auf Internet gibt, bedarf es der Vergewisserung und Eingrenzung, was das Internet in rechtlicher Hinsicht überhaupt ist. Technisch ist das Internet nämlich ohne weiteres reproduzierbar, ${ }^{7}$ in administrativer Hinsicht hat es große Wandlungen durchgemacht, ohne aber seinen eigentlichen Charakter zu verändern. ${ }^{8}$ Entscheidendes Spezifikum für die rechtliche Einordnung des Internets ist, dass es sich um ein umfassendes globales Netzwerk handelt. Die Teilnahme ist (größtenteils) freiwillig, jedenfalls sind die staatlichen und amtlichen Seiten nicht strukturprägend. Und dieses Spezifikum der Freiwilligkeit ${ }^{9}$ ist auch der Grund, warum das Internet zwar da ist, aber nicht geschaffen werden kann.

3 F.C. Mayer, Das Internet, das Völkerrecht und die Internationalisierung des Rechts, ZfRSoz 2002, S. 93 (105); A. Segura-Serrano, Internet Regulation and the Role of International Law, Max Planck Yearbook of United Nations Law X (2010), S. 191 (267); aus Perspektive der Meinungsfreiheit $H$. Schulze-Fielitz, in: H. Dreier (Hrsg.), Grundgesetzkommentar, Bd. I, 2. Aufl., Tübingen 2004, Art. 5 I, II Rn. 9; umfassend M. Gets, Meinungsäußerungs- und Informationsfreiheit im Internet aus Sicht des Völkerrechts, Berlin 2002, S. 53 ff., zusammenfassend dann S. $186 \mathrm{ff}$.

4 S. LSG Essen, Beschl. v. 23.4.2010, Az. L 6 AS 297/10 B, Tz. 7 (juris); LSG München, Beschl. v. 29.1.2010, Az. L 7 AS 41/10 B ER, Tz. 15 (juris); differenzierend LSG Celle NdsVBl. 2003, S. 269 (269 f.) (sozialrechtlicher Anspruch von Schülern auf PC).

5 BAG NJW 2010, S. 1901 (1901 ff.); zum Anspruch des einzelnen Betriebsratsmitglieds BAG RDV 2010, S. 227 (Ls. 1).

6 BVerfGE 120, S. 274 (304) - Online-Durchsuchung; ähnlich die Abschlusserklärung des Treffens der für Medien zuständigen Minister der Mitgliedstaaten des Europarat am 28. u. 29.5.2009 in Reykjavik.

7 Dies ist weniger spektakulär, als es auf den ersten Blick klingt. Denn das Zusammenschalten von Rechnern und elektronischen Geräten basiert heute oft auf dem Internetprotokoll TCP/IP. Insoweit ist jedes Intranet und jede Heimvernetzung eine Reproduktion des Internets.

8 Für den Zweck einer Einführung und geschichtlichen Grundlegung kann auf V. Géczy-Sparwasser, Die Gesetzgebungsgeschichte des Internets, Berlin 2003, und J. Abbate, Inventing the Internet, Cambridge, Mass.; MIT Press 1999, verwiesen werden.

9 G. Nolte, Das Verfassungsrecht vor den Herausforderungen der Globalisierung, VVDStRL LXVII (2008), S. 127 (144), sprich von der „globalisierungstypischsten Freiheit“. 
Keine Macht der Welt - keine nationale, keine totalitäre und auch keine internationale - vermag die umfassende Beteiligung und Vernetzung zu erzwingen, die das Internet ausmachen. Auch kann keine Macht der Welt - außer vielleicht die USA ${ }^{10}$ - das Internet ausschalten. Regulieren lässt es sich von den nationalstaatlichen Rechtsordnungen immer nur unvollkommen.

Allerdings sind die zentralen Voraussetzungen des Internets nationalstaatlichen Regelungen durchaus zugänglich. Dies ist zum einen in technischer Hinsicht die herkömmliche Telekommunikationsinfrastruktur (B.) und zum anderen der freie Fluss von Information (C.). ${ }^{11}$

\section{B. TK-Infrastruktur als Voraussetzung des Internets}

\section{Gewährleistung einer TK-Infrastruktur}

Dass in Deutschland eine telekommunikative Infrastruktur besteht, hat der Staat in concreto: der Bund ${ }^{12}$ - zu gewährleisten. Art. $87 \mathrm{f} \mathrm{Abs.} 1$ GG schreibt dies mit der Maßgabe „angemessener und ausreichender Dienstleistungen“ vor. Auf europäischer Ebene „trägt die Union zum Auf- und Ausbau transeuropäischer Netze [im Bereich der] Telekommunikation " bei (Art. 170 Abs. 1 AEUV). ${ }^{13}$

Was „angemessen und ausreichend“ ist bzw. in welchem Maße ein „Auf- und Ausbau" stattfindet, ist zwar methodisch autonom aus der Verfassung bzw. dem Primärrecht abzuleiten. Tatsächlich aber findet die Konkretisierung dieser Rechtsbe-

10 S. zum Entwurf eines „Protecting Cyberspace as a National Asset Act“ (PCNAA) C. Kurz, Sicherheit im Netz? Nur durch Vernetzung!, F.A.Z. v. 6.8.2010, S. 31.

11 Diese beiden Strukturmerkmale, diese Unterscheidung zwischen nachrichtentechnischer Übertragung und Inhalt, sind - vielleicht überraschenderweise - auch im Grundgesetz angelegt. Es unterscheidet nämlich im Medien- und Kommunikationsbereich seit jeher zwischen der Übertragung von Inhalten und den Inhalten selbst. Diese Unterscheidung hat ihren Ursprung zunächst in der föderalen Gesetzgebungsverteilung: Der Bund war und ist für das Fernmeldewesen bzw. heute die Telekommunikation zuständig (Art. 73 Abs. 1 Nr. 7 GG) und die Länder für den Rundfunk (vgl. Art. 23 Abs. 6 S. 1 Var. 3 GG). Doch diese Abschichtung enthält nicht nur eine föderale Logik, sondern auch eine in der Sache selbst liegende Wahrheit. So unterscheidet die Telekommunikationstechnik in Gestalt von Protokollebenen (TCP/IP, ISO-OSI) verschiedene Schichten. Und diese Unterscheidung in Schichten ist dann auch für das deutsche Internetrecht (TKG - TMG/RStV) und - etwas anders - für das deutsche Datenschutzrecht $(\mathbb{S} 88 \mathrm{ff}$. TKG - $\mathbb{S} 11 \mathrm{ff}$. TMG - BDSG) prägend (zu den föderalen Aspekten des IuKDG v. 22.7.1997 (BGBl. I S. 1870), der ersten deutschen Internetgesetzgebung, Géczy-Sparwasser, Gesetzgebungsgeschichte (Fn. 8), S. 194-206).

12 Allerdings sind teilweise, etwa bei einer Unterversorgung mit Breitbandanschlüssen, auch die Gemeinden aufgerufen, in Wahrnehmung der „Angelegenheiten der örtlichen Gemeinschaft" (Art. 28 Abs. 1. S. 1 GG), Telekommunikation zu gewährleisten, was aber wegen der Vorgabe des Art. 87 f Abs. 2 S. 1 GG nur durch formell privatrechtliche Kommunalunternehmen geschehen kann (zum Streitstand K. Windthorst, in: M. Sachs (Hrsg.), Grundgesetz, 5. Aufl., München 2009, Art. 87 f Rn. 28 a).

13 Daneben macht die Europäische Union durch den Telekommunikations-Rechtsrahmen zur Verwirklichung des Binnenmarkts selbstverständlich auch Vorgaben für die Gestaltung und den Ausbau der Telekommunikationsnetze in den Mitgliedstaaten. 
griffe sekundärrechtlich durch die Universaldienstrichtlinie ${ }^{14}$ bzw. einfachgesetzlich durch $\mathbb{S} \mathbb{S} 78-87$ TKG und das Post- und Telekommunikationssicherstellungsgesetz ${ }^{15}$ statt.

Gegenwärtig umfassen diese Vorgaben auf der Ebene des deutschen Rechts das Internet nicht ausdrücklich. Vielmehr bezieht sich die Universaldienstleistungsverpflichtung nach $\mathbb{7} 78$ Abs. 2 Nrn. 2-5 TKG nur auf das gedruckte Telefonbuch, die Telefonauskunft, die Telefonzelle und die Notrufmöglichkeit. Von $\mathbb{\$} 78$ Abs. 2 Nr. 1 TKG ist die Fernkommunikation nur in Form der ortsgebundene Sprachtelefonie, also des klassischen Telefonanschlusses, vorgesehen. Allerdings ist hierüber mittels eines Modems jedenfalls ein schmalbandiger Internetzugang möglich. ${ }^{16}$ Ein Breitbandanschluss ist dagegen ebenso wenig wie ein mobiler Internetanschluss Teil der einfachgesetzlichen Grundversorgung, da keine Universaldienstleistung. Diese nur rudimentären Vorgaben auf dem Stand der neunziger Jahre des vergangenen Jahrhunderts werden aufgrund Europarechts auch in den nächsten Jahren nur unwesentlich ausgeweitet (Art. 4 Abs. 2 Hs. 1 RL 2002/22/EG i.d.F. d. RL 2009/136/ EG): Den Mitgliedstaaten wird durch die Novellierung des europäischen TK-Rechtsrahmens lediglich die Gewährleistung eines „funktionalen Internetzugangs“ vorgegeben, was zudem unter dem Vorbehalt der „technischen Durchführbarkeit“ steht. Hiermit wird einerseits immerhin eine dynamische Komponente eingeführt, bedeutet andererseits aber auch nur eine graduelle Verbesserung zum bislang schon gewährleisteten Modemanschluss. ${ }^{17}$

Aus anderen Vorschriften des Grundgesetzes könnte allerdings - jedenfalls in methodischer Hinsicht - auch ein höheres Diensteniveau abgeleitet werden. Argumentativ müsste dies durch den Rückgriff auf die Figur der grundrechtlichen Schutz-

14 Richtlinie 2002/22/EG v. 7.3.2002 (ABl. EG L 108 S. 51) i.d.F. d. Richtlinie 2009/136/ EG v. 25.11.2009 (ABl. EU L 337 S. 11). - Zu Universaldiensten als einer „Bauform“ der Gewährleistungsverwaltung C. Franzius, Gewährleistung im Recht, Tübingen 2009, S. 566 ff.

15 Gesetz zur Sicherstellung des Postwesens und der Telekommunikation v. 14.9.2004 (BGBl. I S. 2325, ber. S. 2378). - Ob hiervon auch das Internet als Ganzes umfasst ist, ist umstritten (Chr. Möllers/L. Pflug, Verfassungsrechtliche Rahmenbedingungen des Schutzes kritischer Infrastrukturen, in: M. Kloepfer (Hrsg.), Schutz kritischer Infrastrukturen, Baden-Baden 2010, S. 47 (53) zu $\$ 3$ Abs. 1 Nr. 15 BSIG).

16 Abhängig ist die Modemnutzung von der Einhaltung der Vorgaben des FTEG (Th. Krull/M. Klümper, Zulassung von Telekommunikationsendeinrichtungen und Funkanlagen, in: M. Wissmann (Hrsg.), Telekommunikationsrecht, 2. Aufl., Heidelberg 2006, Kap. 11 Rn.11; vgl. VG Köln GewArch. 2008, 90 ff.), früher der BZT-, davor der ZZF- und wieder davor der FTZ-Zulassung (vgl. etwa KG GRUR 1991, S. 690 (691); P. M. Rütter, Unerlaubte Anschließung nicht zugelassener Endeinrichtungen an das öffentliche Telekommunikationsnetz, NJW 1990, S. 1586 f.; zum europarechtlichen Hintergrund F. Hochbaum/R. Klotz, in: H. v. d. Groeben/J. Schwarze (Hrsg.), Kommentar zum EU-/EG-Vertrag, 6. Aufl., Baden-Baden 2003, Art. 86 EGV Rn. 128ff.).

17 Andere Mitgliedstaaten, z.B. Finnland (s. F.A.Z. v. 23.7.2010, S. 31), sind hinsichtlich des Breitbandzugangs auf der Ebene ihres nationalen Rechts schon weiter. 
pflichten begründet werden. ${ }^{18}$ Relevanter ist aber derzeit die unterschiedlich gute und schnelle Versorgung mit Breitbanddiensten zwischen Stadt und Land, ${ }^{19}$ die man unter dem Stichwort der Einheitlichkeit der Lebensverhältnisse problematisieren kann.

\section{Gewährleistung eines TK-Zugangs}

Die Gewährleistung der dem Internet zugrundeliegenden Infrastruktur ist rechtlich zu unterscheiden von der Möglichkeit des individuellen Zugangs hierzu.

\section{Zugangsmöglichkeit}

Der Anschluss an das Telefonnetz und damit auch an das Internet ist im deutschen Recht als Kontrahierungszwang der zu Universaldienstleistung verpflichteten Unternehmen ausgestaltet ( $\$ 84$ Abs. 1 TKG). Niemand kann deshalb von der Teilnahme am Telekommunikationsnetz ausgeschlossen werden. Hierbei handelt es sich strukturell um ein gewährleistungsrechtliches Diskriminierungsverbot. ${ }^{20}$ Unausgesprochen beinhaltet dies auch das Verbot einer Zugangsregulierung (auf technischer Ebene) und damit eine Ausprägung einer allgemeinen Internetfreiheit.

\section{Leistungsrechtlicher Anspruch auf Internetzugang}

Doch ist die diskriminierungsfreie telekommunikative Zugangsmöglichkeit keine hinreichende Bedingung für einen tatsächlichen Zugang zum Internet. Für einen solchen muss nämlich ein Vertrag über den Netzanschluss geschlossen werden, und es bedarf eines Datenendgeräts; beides kostet Geld.

Für den Zugang zum Internet muss der Marktpreis gezahlt werden. ${ }^{21}$ Die finanziellen Mittel hierzu werden bei der Berechnung der Sozialhilfe und daran angelehnt auch des Sozialgelds und des Arbeitslosengelds II berücksichtigt. Nach $\mathbb{} 2$ Abs. 2

18 M. Schmidt-Preuß, Verwaltung und Verwaltungsrecht zwischen gesellschaftlicher Selbstregulierung und staatlicher Steuerung, VVDStRL LVI (1997), S. 160 (172 m.w.N.); W. Hoffmann-Riem, in: E. Denninger/W. Hoffmann-Riem/H.-P. Schneider/E. Stein (Hrsg.), Alternativkommentar, 3. Aufl., Baden-Baden Stand 2001, Art. 5 GG, Rn. 112, mit Verweis auf R. Wendt, in: I. v. Münch/P. Kunig (Hrsg.), Grundgesetz-Kommentar, Bd. 3, 5. Aufl., München 2000, Art. 5 GG, Rn. 28.

19 Rechtspolitisch hierzu z.B. B. Holznagel/S. Deckers/M. Schramm, Erschließung des ländlichen Raums mit Breitband - die Leitlinien der Kommission zum Breitbandausbau, NVwZ 2010, S. 1059 ff.

20 Hiervon zu unterscheiden ist die wettbewerbsrechtliche und gleichheitsrechtliche Nicht-Diskriminierung bei der (Netz-)Zusammenschaltung, wie sie nicht nur im TK-Sektor problematisch sein kann, sondern auch in anderen Bereichen (z.B. Presse-Grosso, Barsortimente, Pharmagroßhandel; vgl. $\mathbb{S} 19$ Abs. 4 Nr. 4 GWB).

21 Allerdings bietet die Deutsche Telekom, der (gegenwärtige) Universaldienstanbieter, (freiwillig) einen Sozialtarif (in Form einer Gutschrift) an, so dass tatsächlich sogar weniger als der Marktpreis gezahlt werden muss (vgl. die „Allgemeinen Bedingungen Sozialtarif“ der Telekom Deutschland GmbH). 


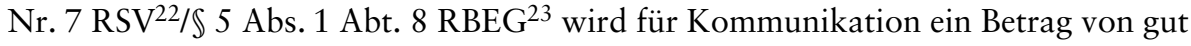
$30 €$ (für einen Alleinstehenden) zugrundegelegt. Dadurch kann ohne weiteres sogar ein (kleinerer) Flatrate-Anschluss realisiert werden.

Der Anschluss alleine verhilft noch nicht ins Netz. Hierfür bedarf es Hardware, Software und Know-how. Dies zu beschaffen überlässt der Staat dem Einzelnen. Software (Browser usw.) ist kostenlos verfügbar und das technische Wissen dank Anleitungen in einer Weise aufbereitet, dass auch technisch Unbewanderte einen Internet-Anschluss installieren können. ${ }^{24}$ Doch die Hardware (Computer, Router/Modem) kostet auch nach dem Preisverfall der vergangenen Jahre immer noch einen mittleren dreistelligen Betrag. Er ist vom Einzelnen selbst aufzubringen; die soziale Grundsicherung umfasst bislang einen Computer (noch) nicht; ${ }^{25}$ auch zukünftig wird er (nur) durch Ansparen anschaffbar sein. ${ }^{26}$ - Das Zwangsvollstreckungsrecht ist in diesem Bereich schon etwas weiter: Nach $\mathbb{S} 811$ Abs. 1 Nr. 1 ZPO unterliegt ein PC samt Zubehör nicht der Zwangsvollstreckung, wenn er dem persönlichen Gebrauch dient, nach $\ 811$ Abs. 1 Nr. 5 ZPO ist die Zwangsvollstreckung ausgeschlossen, wenn ein Rechner Voraussetzung für die Fortsetzung einer Berufs- oder Erwerbstätigkeit ist. $^{27}$ Gegebenenfalls muss aber der Vollstreckungsschuldner eine Austauschpfändung nach $\$ 811$ a ZPO dulden. ${ }^{28}$

Insoweit ist für das einfachgesetzliche Recht in Deutschland gegenwärtig eine bemerkenswerte Zwischen- oder besser: Übergangssituation zu konstatieren. Ein schmalbandiges Internet ist Teil der telekommunikativen Grundversorgung. Die Kosten für den Zugang zum Internet werden als Teil der sozialen Grundsicherung berücksichtigt, die hierfür erforderliche Hardware allerdings nicht. Besitzt (und benutzt) man jedoch die erforderlichen Geräte, sind sie pfändungsfrei.

22 Regelsatzverordnung v. 3.7.2004 (BGBl. I S. 1067), zul. geänd. d. Art. 17 G v. 2.5.2009 (BGBl. I S. 416); Aufhebung geplant d. Art. 12 Abs. 1 des Entwurfs eines Gesetzes zur Ermittlung von Regelbedarfen und zur Änderung des SGB II und SGB XII (BT-Drucks. 17/3404), das zum Zeitpunkt der Drucklegung dieses Beitrags noch im Vermittlungsausschuss beraten wurde.

23 Regelbedarfs-Ermittlungsgesetz (Art. 1 des Entwurfs eines Gesetzes zur Ermittlung von Regelbedarfen und zur Änderung des SGB II und SGB XII (Fn. 22)).

24 Personifiziert wurde dies durch den damaligen Tennisspieler Boris Becker, dessen „Bin ich schon drin, oder was? “ so verbreitet war, dass auch Konkurrenten diesen Werbespruch aufgriffen (vgl. LG München I ZUM-RD 2000, S. 549 ff.).

25 LSG Essen, Beschl. v. 23.4.2010, Az. L 6 AS 297/10 B, Tz. 9 (Juris); LSG München, Beschl. v. 29.1.2010, Az. L 7 AS 41/10 B ER, Tz. 15 (Juris).

26 Vgl. BT-Drucks. 17/3404, S. 51.

27 Die Rechtsprechung hierzu ist uneinheitlich (für einen Pfändungsschutz AG Bersenbrück DGVZ 1990, S. 78; LG Heilbronn MDR 1994, S. 405; AG Essen DGVZ 1998, S. 94; dagegen AG Heidelberg DGVZ 1989, S. 15; AG Kiel JurBüro 2004, S. 334 (334 f.)), was aber weniger mit unterschiedlichem lokalen Gerichtsgebrauch zu tun hat, sondern vielmehr auf die besondere Zeit- und Situationsgebundenheit der Zwangsvollstreckung zurückzuführen ist.

28 Speziell zu TK-Endgeräten J.M. Schmittmann, Zur Pfändbarkeit von Telekommunikationsendgeräten, DGVZ 1994, S. 49 (51). 
Der verfassungsrechtlich, vor allem über das Sozialstaatsprinzip gebotenen, aber auch in Art. $87 \mathrm{f} \mathrm{GG} \mathrm{zum} \mathrm{Ausdruck} \mathrm{kommenden} \mathrm{Pflicht} \mathrm{zur} \mathrm{Kommunikationsgrund-}$ versorgung ${ }^{29}$ wird aber gleichwohl (noch) genügt. Denn der Zugang zum Internet muss nicht zwingend von daheim und mit eigenem Rechner hergestellt werden können, sondern der Betroffene kann auch auf den Besuch eines Internet-Cafés verwiesen werden. Daneben hat er die Möglichkeit, Mittel für einen eigenen Rechner anzusparen. Besonderer Berücksichtigung bedarf freilich die Rolle, die das Internet für die schulische und soziale Entwicklung von Kindern spielt. ${ }^{30}$ Auf die spezifischen Bedürfnisse von Kindern hatte das BVerfG in der Hartz IV-Entscheidung ausdrücklich hingewiesen. ${ }^{31}$

Ein eigener Internet-PC ist auch außerhalb des sozialrechtlichen Kontexts im Hinblick auf die staatsbürgerlichen und rechtstaatlichen Rechte nicht geboten. Denn für deren Ausübung und Wahrnehmung ist das Internet (noch) keine Voraussetzung. Die Wahl findet nach wie vor mittels Wahlzettels statt, möglicherweise auch als Briefwahl. Sofern Wahlcomputer überhaupt eingesetzt werden (dürfen), ${ }^{32}$ muss der Wahlberechtigte im Wahllokal anwesend sein. ${ }^{33}$ Auch die Gesetze werden konventionell in einem papierenen Gesetzblatt verkündet. ${ }^{34}$ Soweit elektronische Gerichtsakten $(\$ 298$ a ZPO) geführt werden, betrifft dies nur den Gerichtsbinnenbereich.

Diese sozial(verfassungs)rechtliche Bewertung muss sich aber in dem Maße ändern, in dem der Anschluss an das Telekommunikationsnetz und darauf basierend auch an das Internet rechtlich vorausgesetzt wird. ${ }^{35}$ Auch würde eine andere Beurteilung geboten sein, wenn das mobile Internet, also die ortsabhängige Nutzung von Kom-

29 Hierzu auch J. Wieland, in: H. Dreier (Hrsg.), Grundgesetz-Kommentar, Bd. III, 2. Aufl., Tübingen 2008, Art. 87 f Rn. 17 m.w.N.

30 Vgl. LSG Celle NdsVBl. 2003, S. 269 (269 f.).

31 BVerfGE 125, S. 175 (231) - Hartz IV.

32 Vgl. BVerfGE 123, S. $39 \mathrm{ff}$.

33 Dies ist in den $\mathbb{S} 31 \mathrm{ff}$. BWahlO nicht ausdrücklich bestimmt, allerdings als Selbstverständlichkeit von diesen Normen erkennbar vorausgesetzt (vgl. $\$ 9$ BWahlGV zur Wahlzelle bei der Verwendung von Wahlcomputern) - Zum verfassungsrechtlichen Rahmen demokratischer Teilhabehandlungen im Internet R. Zimmermann, Wahlen, Abstimmungen und Petitionen im Internet vor den Schranken des Grundgesetzes, in: H. Goerlich (Hrsg.), Rechtsfragen der Nutzung und Regulierung des Internet, Berlin 2010, S. $183 \mathrm{ff}$.

34 Vgl. hierzu Art. 102 S. 2 saarVerf., der eine elektronische Verkündung von Gesetzen (nach Maßgabe eines noch zu erlassenden Gesetzes) zulässt (A. Guckelberger, Der Übergang zur (ausschließlichen) elektronischen Gesetzesverkündung, Baden-Baden 2009; kritisch Chr. Gröpl, in: R. Wendt/R. Rixecker (Hrsg.), Verfassung des Saarlands, Saarbrücken 2009, Art. 102 Rn. 10). - Rechtstatsächlich ist jedenfalls die Tendenz zu beobachten, dass angesichts der elektronischen Verfügbarkeit von konsolidierten Normtexten die Neuverkündungen abnehmen (vgl. zur Lage in NRW A. Becker/A. Poll-Wolbeck, Zur Gesetzgebungspraxis in Nordrhein-Westfalen, DÖV 2010, S. 560 (560)).

35 Soweit ersichtlich, ist dies bislang jedoch nur für unternehmerische Tätigkeiten der Fall. Verwiesen werden kann hierfür beispielhaft auf die verschiedenen steuerrechtlichen Verpflichtung zur Abgabe von Erklärungen in elektronischer Form (z.B. $\mathbb{1} 180$ Abs. 1 S. 1 UStG, $\mathbb{} 5$ b EStG), auf die berufsrechtliche Pflicht von Anwälten, eine Telefon oder andere Telekommunikationsmittel vorzuhalten (dazu K. v. Lewinski, Grundriss des Anwaltlichen Berufsrechts, 2. Aufl., Baden-Baden 2008, S. 142), und aus dem Bereich des Umweltrechts etwa auf $\$ 4$ Abs. 4 TEHG. 
munikationsmöglichkeiten, eine allgemeine gesellschaftliche Bedeutung bekommen hat.

\section{Abwehrrechtlicher Schutz}

Neben der Frage der Zugangsermöglichung steht die der (hoheitlichen) Zugangsbeschränkung. Wie weit kann der Staat einen bestehenden Telekommunikationszugang beschränken und sperren?36

\section{Nur randständiger Schutz durch technikbezogene Grundrechte}

Bemerkenswerterweise sind die in dem Zusammenhang allgemein mit der Telekommunikation am ehesten assoziierten Grundrechte - das Fernmeldegeheimnis (Art. 10 Abs. 1 GG), das „Recht auf Informationelle Selbstbestimmung“ und das erst vor kurzem entdeckte ${ }^{37}$ Computer-Grundrecht (Grundrecht auf Gewährleistung der Vertraulichkeit und Integrität informationstechnischer Systeme, GRaGVIiS ${ }^{38}$ ) nicht bzw. nur am Rande einschlägig. ${ }^{39}$ So schützt das Fernmeldegeheimnis nicht den Zugang zur Telekommunikation, sondern setzt ihn vielmehr voraus. Auch das (grundrechtliche) Datenschutzrecht kommt erst zur Anwendung, wenn Daten vom Staat erhoben bzw. gespeichert werden. Schließlich betrifft auch das GRaGVIiS sachlich nicht den Zugang zu Kommunikationsinfrastruktur, sondern (nur) die technische Gerätschaft hierfür.

\section{Allgemeine Handlungsfreiheit}

Allgemein ist der Zugang zum Telekommunikationsnetz grundrechtlich also nicht speziell, sondern nur als Teil der allgemeinen Handlungsfreiheit (Art. 2 Abs. 1 GG)

36 Dies ist im Jahre 2010 v.a. im Zusammenhang mit Internetsperren für Urheberrechtsverletzer diskutiert worden (sog. Three Strikes-Regelung, z.B. die nach der zuständigen Haute Autorité pour la Diffusion des Euvres et la Protection des Droits sur Internet benannte loi Hadopi (s. hierzu die beiden Entscheidungen des französischen Verfassungsrats (Conseil Constitutionnel) v. 20.6. 2009, Az. 2009-580 DC und v. 22.10.2009, Az. 2009-590) oder eine zwischenzeitlich diskutierte Reglungsoption im ACTA [Anti-Counterfeiting Trade Agreement]) (dazu H. Greve/F. Schärdel, Three strikes and you're out! Internetsperren wegen Urheberrechtsverstößen, ZRP 2009, S. 54 ff.; A. Metzger, A Primer on ACTA: What Europeans should fear about the Anti-Counterfeiting Trade Agreement, JIPITEC I (2010), S. 109 (114)).

37 Weitere „Neuentdeckungen“ in diesem Bereich sind das „Grundrecht auf IT-Abwehr“ (D. Heckmann, Grundrecht auf IT-Abwehr? Freiheitsrechte als Abwehrrechte gegen aufgedrängtes E-Government, MMR 2006, S. 3 ff.) und das „Grundrecht auf Verschlüsselung“ (vgl. J. Gerhards, (Grund-)Recht auf Verschlüsselung?, Baden-Baden 2010).

38 Hierzu Chr. Herrmann, Das Grundrecht auf Gewährleistung der Vertraulichkeit und Integrität informationstechnischer Systeme, Frankfurt 2010.

39 Die genannten grundrechtlichen Gewährleistungen können hinsichtlich des Zugangs zu Telekommunikationssystemen allerdings gleichwohl, dann aber nur indirekt relevant sein. Denn sie schützen den Einzelnen jedenfalls mittelbar vor der Befangenheit, die von staatlichen Überwachungs-, Speicherungsund Manipulationseingriffen ausgehen können. So ist etwa die für manche Arten der Internetfilterung eingesetzte Deep Packet Inspection an Art. 10 GG zu messen. Der Einsatz einer wie in China angedachten verpflichtenden Filtersoftware auf den einzelnen Rechnern in Deutschland wäre ebenfalls grundrechtsrelevant. Und schließlich hat auch die (in Deutschland vorerst aufgeschobene und aufgehobene) Vorratsdatenspeicherung grundrechtsrelevante Auswirkungen auf die Zugangsfreiheit zu Telekommunikationsnetzen. 
geschützt; im beruflichen bzw. unternehmerischen Kontext kommen daneben noch Art. 12 bzw. Art. 14 GG in Betracht. Allgemein kann so auf der Grundlage eines (im übrigen verhältnismäßigen) Gesetzes die Nutzung von Telekommunikationseinrichtungen $^{40}$ und damit auch des Internets beschränkt bzw. verboten werden. Solche Eingriffe sind freilich selten. Ein Beispiel hierfür sind die Beschränkungen für Strafgefangene, Telekommunikationsnetze und damit auch das Internet zu nutzen (vgl. $\$ 28$ u. $\$ 32$ StVollzG sowie den Vorbehalt des $\$ 70$ Abs. 2 Nr. 2 StVollzG ${ }^{41}$ ). Zu den strafrechtlichen Sanktionen zählt eine Internetsperre (noch) nicht. ${ }^{42}$ Polizeirechtlich wäre ein Verbot der Internetnutzung (auf der Grundlage der Generalklausel) zwar denkbar, praktische Konstellationen sind allerdings nur schwer vorstellbar.

\section{Zwischenergebnis}

Hinsichtlich des technischen Zugangs zum Internet und seiner telekommunikativen Nutzung stehen starke objektivrechtliche Verfassungsgehalte einem nur grundlegenden abwehrrechtlichen Schutz gegenüber. Auf normativer Ebene fehlt es jedenfalls an einem (speziellen) Grundrecht.

Diese auf den ersten Blick möglicherweise unzureichend erscheinende Rechtslage modifiziert sich allerdings noch, soweit nämlich der technische Zugang zum Internet Voraussetzung und damit Grundrechtsvoraussetzung für andere verfassungsrechtlich geschützte (kommunikative und informationelle) Verhaltensweisen ist. ${ }^{43}$ Dies leitet über zu der Frage, wie weit ein auf Inhalte und Information bezogenes „Recht auf Internet" besteht.

\section{Verbreitung von und Zugang zu Inhalten im Internet}

\section{Gewährleistungsgehalte}

Auch wenn die inhaltliche Nutzung und Teilnahme am Internet ebenso wie der telekommunikative, technische Zugang in der Verfassung nicht unmittelbar angesprochen wird, ist leicht erkennbar, dass das Grundgesetz den Informationsaustausch voraussetzt. Fraglich ist aber, ob der Staat den freien Informationsfluss auch im Internet gewährleisten muss, soweit er etwa für die demokratische Willensbildung, die

$40 \mathrm{Zu}$ einem Pilotversuch in Hessen, in Gefängnissen „Handy-Blocker“ einzusetzen, F.A.Z. v. 23.6.2010, S. 4.

41 Hierzu OLG Celle NStZ 2002, S. 111 (112); F. Arloth, StVollzG, 2. Aufl., München 2008, $\mathbb{} 32$ Rn. 1; R.-P. Calliess/H. Müller-Dietz, Strafvollzugsgesetz, 11. Aufl., München 2008, $\$ 32$ Rn. 3; Schulze-Fielitz (Fn. 3), Art. 5 I, II Rn. 186; umfassend F. Knauer, Strafvollzug und Internet, Berlin 2006; s. auch O. M. Köppen, Das Grundrecht der Informationsfreiheit unter besonderer Berücksichtigung der neuen Medien, Köln 2004, S. 193-199.

42 S. nur J. Baumann/U. Weber/W. Mitsch, Strafrecht Allgemeiner Teil, 11. Aufl., Bielefeld 2003, S. $778 \mathrm{ff}$.

43 Vgl. BVerfGE 90, S. 27 ff. - Parabolantenne; s. aus europarechtlicher Perspektive A. Ferti, The Internet Freedom Provision of the EU Telecoms Package, CRi 2010, S. 1 (8); M. Kloepfer, Entstehenssicherung und Bestandsschutz, München 1970, S. 17, spricht von der „Hintereinanderschaltung von Grundrechten“. 
Persönlichkeitsentfaltung, wirtschaftliche Tätigkeit und die gesellschaftliche Teilhabe notwendig ist.

\section{Gewährleistung der demokratischen Willensbildung}

Die „politische Willensbildung des Volkes“, wie sie in Art. 21 Abs. 1 S. 1 GG angesprochen ist, leitet sich unmittelbar vom Demokratieprinzip her. Eine Herrschaft des Volkes ist ohne die Möglichkeit, dass sich das Volk, die Allgemeinheit, einen politischen Willen bildet, nicht denkbar. ${ }^{44}$ Das Bundesverfassungsgericht bezeichnet die Meinungsfreiheit denn auch als für die Demokratie schlechthin konstituierend. ${ }^{45}$ Den (Stimm-)Bürgern muss es möglich sein, Informationen und Meinungen einzuholen und zu verbreiten. Vor dem Hintergrund der Erfahrungen vieler europäischer Völker mit totalitären Regimen und mit fremder Besatzung sowie dem aufkommenden Informationszeitalter wird der freie Informationszugang, oft verkürzt Informationsfreiheit genannt, immer wichtiger. ${ }^{46}$

In einer freibeitlichen demokratischen Grundordnung muss die Meinungsbildung aber grundsätzlich dem Meinungsmarkt überlassen bleiben. Insoweit gibt es keine Befugnis des Staates zur Gestaltung der Meinungslandschaft. ${ }^{47}$ Vielmehr ist zu bedenken, dass jeder (Gewähr-)Leistungseingriff in die Meinungsbildung immer auch einen Eingriff in den Kommunikationsprozess selbst bedeutet. ${ }^{48}$ Dies gilt nicht nur für den klassischen Fall der Pressesubventionen, ${ }^{49}$ sondern auch für das Subventionieren von (abgrenzbaren) Nutzergruppen (z.B. Hartz IV-Empfänger oder Studenten), da sich - der Marktlogik folgend - das Angebot auf dem Meinungsmarkt entsprechend anpasst und verändert.

Von dem Eingriff in den Meinungsmarkt kann die Regelung des Medienmarkts unterschieden werden. Hier kann zumindest eine Befugnis zur Gestaltung der Medienlandschaft leichter begründet werden. ${ }^{50}$ Wie erwähnt, wird die Presse- ${ }^{51}$ und Rund-

44 BVerfGE 89, S. 155 (185) - Maastricht: öffentliche Meinung als „vorrechtliche Voraussetzung“ der Demokratie.

45 St. Rspr., s. BVerfGE 20, S. 56 (97), mit Verweis auf BVerfGE 7, S. 198 (208); in der Sache auch schon BVerfGE 5, S. 85 (134 f., 205); ferner BVerfGE 101, S. 361 (389); aus dem Schrifttum statt aller $H$. Bethge, in: M. Sachs (Hrsg.), Grundgesetz, 5. Aufl., München 2009, Art. 5 Rn. 17.

46 Vgl. Schulze-Fielitz (Fn. 3), Art. 5 I, II Rn. 35.

47 Chr. Starck, in: H. v. Mangoldt (Begr.)/F. Klein/ders. (Hrsg.), Kommentar zum Grundgesetz, Bd. 1, 6. Aufl., München 2010, Art. 5 Rn. 6.

48 Starck (Fn. 47), Art. 5 GG, Rn. 20.

49 Zum deswegen bestehenden Gesetzesvorbehalt OVG Berlin NJW 1975, S. 1938 (1940); OLG Frankfurt am Main NVwZ 1993, S. 706 (707); P. Lerche, Verfassungsrechtliche Fragen der Pressekonzentration, Berlin 1974, S. $105 \mathrm{f}$.

50 W. Hoffmann-Riem, Kommunikations- und Medienfreiheit, in: E. Benda/W. Maihofer/H.-J. Vogel (Hrsg.), Handbuch des Verfassungsrechts, 2. Aufl., Berlin 1994, S. 191 (195).

51 BVerfGE 20, S. 162 (174); dazu M. Bullinger, Freiheit von Presse, Rundfunk, Film, in: J. Isensee/P. Kirchhof (Hrsg.), Handbuch des Staatsrechts, Bd. 7, 3. Aufl., Heidelberg 2009, $\mathbb{1 6 3}$ Rn. 36 ff., der demgegenüber den allgemeinen Schutz der Pressefreiheit auch auf sonstige Druckerzeugnisse betont. 
funkfreiheit ${ }^{52}$ (auch) in ihrer objektivrechtlichen Ausprägung seit jeher als unerlässlich angesehen. Diese beiden Medien waren schon in der Zeit vor dem Internet Gegenstand eines intensiven Gewährleistungsdiskurses. Hinsichtlich des Internets gibt es ähnliche Begründungen. ${ }^{53}$ Doch bedeuten die strukturelle Pluralität des Internets und der nur minimale Mitteleinsatz, der erforderlich ist, dort Meinungen zu verbreiten, dass ein staatliches Wächteramt hinsichtlich der Online-Meinungsfreiheit gegenwärtig nicht aktiv ausgeübt werden muss und darf. ${ }^{54}$

\section{Gewährleistung der Persönlichkeitsentfaltung und Wirtschaftstätigkeit}

Die „schlechthin konstituierende“ Bedeutung des Art. 5 GG für eine freiheitliche demokratische Grundordnung darf allerdings nicht verdecken, dass dessen Gewährleistungen nicht auf die politische Kommunikation beschränkt sind. ${ }^{55}$ Speziell in Bezug auf das Internet hat das Bundesverfassungsgericht die Bedeutung auch für die Persönlichkeitsentfaltung herausgestellt. ${ }^{56}$ Und seit jeher ist jedenfalls im Grundsatz anerkannt, dass kommerzielle Kommunikation, also geschäftliche Werbung, dem Schutz des Art. 5 Abs. 1 GG unterfallen kann. ${ }^{57}$ Wirtschaftliche und berufliche Entfaltung über das Internet sind daneben immer auch über Art. 14 bzw. Art. 12 GG geschützt.

Trotz der großen Auswahlfreiheit im Internet sind die Nutzer - das zeigt die Erfahrung - in einer strukturell schwächeren Position, insbesondere hinsichtlich des Datenschutzes. Wegen der Tendenz des Internets zu Anbietermonopolen (sog. Netzef$\mathrm{fekt}^{58}$ ) ist ein Ausweichen auf andere Anbieter oft keine realistische Alternative. Auch

52 BVerfGE 12, S. 205 (260).

53 Starck (Fn. 47), Art. 5 GG, Rn. 165.

54 Ein Vorbote möglicher zukünftiger Gefährdungslagen, die von marktmächtigen Inhalteanbietern und -mittlern ausgehen können, ist die (auch) inhaltliche Auswahl im sog. App Store der Fa. Apple. Bezeichnend ist der (vorübergehende) Ausschluss einer Comic-Fassung von Wildes „Ulysses“ (F.A.Z. v. 17.6.2010, S. 39; zu diesem Problemkreis bereits Mecklenburg, Internetfreiheit (Fn. 2), S. 537).

55 BVerfGE 101, S. 361 (389); a.A. Ridder, Meinungsfreiheit, in: F. L. Neumann/H. C. Nipperdey/U. Scheuner (Hrsg.), Die Grundrechte, Bd. 2, Berlin 1954, S. 243 (257 ff.).

56 Vgl. BVerfGE 120, S. 274 (303) - Online-Durchsuchung.

57 BVerfGE 71, S. 162 (175) (jedenfalls hinsichtlich des meinungsbildenden Inhalts); M. Antoni, in: D. Hömig (Hrsg.), Grundgesetz, 9. Aufl., Baden-Baden 2010, Art. 5 Rn. 6.

58 Hierzu A. Picot/R. Neuburger, Wirtschaftliche Grundlagen, in: T. Hoeren/U. Sieber (Hrsg.), Multimedia-Recht, 7. ErgLfg., München Okt. 2003, Rn. 41-43; Chr. Ablborn/D. Seelinger, Business to Business Exchanges: EG-kartellrechtliche Probleme bei Unternehmenskooperationen im Internet, EuZW 2001, S. 552 (555); K. v. Lewinski, Vertraulichkeit und Datenschutz bei B2B-Marktplätzen in: L. Gramlich/D. Kröger/M. Schreibauer (Hrsg.), Rechtshandbuch B2B Plattformen, München 2003, \$16 Rn. 5; zum allg. kartellrechtlichen Netzeffekt D. Zimmer, in: U. Immenga/E.-J. Mestmäcker, Wettbewerbsrecht, Bd. 1, 4. Aufl., München 2007, Art. 81 EGV, Rn. 412 f. 
hat die jüngste Diskussion um Bewertungsportale ${ }^{59}$ gezeigt, dass das Internet neue Gefährdungen für das Persönlichkeitsrecht birgt. Insoweit lässt sich eine Gewährleistungspflicht des Staates und damit eine Schutzpflicht für den Persönlichkeitsschutz herleiten, ${ }^{60}$ dem der Gesetzgeber - bei aller Unvollkommenheit und nationalen Beschränktheit des deutschen Internetrechts - auch nachgekommen ist.

\section{Sozialstaatliche Gewährleistung gesellschaftlicher Teilhabe}

Sozialstaatliche Gewährleistungspflichten in Bezug auf Inhalte des Internets sind dagegen zurzeit eine bloß theoretische Frage. Zwar umfasst das Existenzminimum auch die kulturelle und kommunikative Teilhabe. ${ }^{61}$ Doch würden hieraus erst dann staatlichen Gewährleistungspflichten erwachsen, wenn wesentliche Teile gesellschaftlicher Kommunikation oder des Kulturlebens sich ausschließlich im Internet abspielten $u n d$ nicht kostenlos erreichbar wären. Dies ist gegenwärtig nicht der Fall, so dass auch hier das Wächteramt des Staates ruht.

\section{Keine Gewährleistung des eGovernment}

Ebenfalls kein Teil der grundgesetzlichen Gewährleistung ist die elektronische Verwaltung (eGovernment). ${ }^{62}$ Sie ist von der Verfassung nicht vorausgesetzt, wenngleich inzwischen in ihren Voraussetzungen in Art. 91 c GG zumindest angesprochen. Der Staat muss keine elektronischen Dienstleistungen, ja nicht einmal Informationen im Internet anbieten. Bietet er sie aber an (z.B. im Rahmen des $\ 11$ Abs. 3 IFG), muss er mit Blick auf das Rechtstaatsprinzip und das Gleichheitsgebot sicherstellen, dass niemand hiervon ausgeschlossen bleiben muss. Ist etwa eine Pflicht zur Internetnut-

59 S. hierzu M. Schröder, Persönlichkeitsrechtsschutz bei Bewertungsportalen im Internet, VerwArch 2010, S. 205 ff.; G. Gounalakis/C. Klein, Zulässigkeit von personenbezogenen Bewertungsplattformen, NJW 2010, S. 566 ff.; M. Schütz, Zur Zulässigkeit von Zensuren für Spielerberater und -vermittler im Internet, SpuRt 2010, S. 16 ff. (speziell zu Beratern von Sportlern); Chr. Solmecke, Der Rechtsanwalt auf der Skala von eins bis zehn, NJW-aktuell 2010/10, S. 12 f. (zu Rechtsanwälten); L. Gundermann, Zur datenschutzrechtlichen Zulässigkeit von Bewertungsportalen - am Beispiel des AOK-Arztnavigators, VuR 2010, S. 329 ff. (zu Ärzten); A.-B. Kaiser, Bewertungsportale im Internet - die Spick-mich-Entscheidung des BGH, NVwZ 2009, S. 1474 ff.; grundlegend H. Greve/F. Schärdel, Der digitale Pranger - Bewertungsportale im Internet, MMR 2008, S. 644 ff.

60 Chr. Hohmann-Dennhardt, Freiräume - Zum Schutz der Privatheit, RDV 2008, S. 1 (1 f.); M. Kutscha, Mehr Datenschutz - aber wie?, ZRP 2010, S. 112 (113). - Auch einem grundrechtlichen Schutz der Anonymität scheint das BVerfG nicht ablehnend gegenüberzustehen (vgl. BVerfG NJW 2010, S. 833 (Tz. 258); kritisch hierzu H. A. Wolff, Vorratsdatenspeicherung - Der Gesetzgeber gefangen zwischen Europarecht und Verfassung?, NVwZ 2010, S. 751 (753)).

61 Speziell im Internetkontext S. E. Schulz, Informations- und Kommunikationstechnologie als Grundversorgung, DuD 2010, S. $698 \mathrm{ff}$.

62 S. hierzu G. Britz, Elektronische Verwaltung, in: W. Hoffmann-Riem/E. Schmidt-Aßmann/A. Voßkuhle (Hrsg.), Grundlagen des Verwaltungsrechts, Bd. 2, München 2008, \$26; M. Eifert, Electronic Government, Baden-Baden 2006; J. v. Lucke, Regieren und Verwalten im Informationszeitalter, Berlin 2003. 
zung für Arme unverhältnismäßig, muss über das Bereitstellen konventioneller $\mathrm{Zu}$ gangswege nachgedacht werden. ${ }^{63}$

\section{Keine Gewährleistung einer eCommerce-Infrastruktur}

Schließlich kann auch mit Blick auf die Wirtschaft keine grundgesetzliche Pflicht des Staates festgestellt werden, einen Rahmen für den elektronischen Geschäftsverkehr bereitzustellen. Grundsätzlich ist das Geschäftsleben Sache des Geschäftslebens. Allerdings sind in diesem Bereich zur Zeit auch keine grundlegenden praktischen Probleme verborgen, da der Staat, insbesondere in Umsetzung der eCommerce-Richtlinie, ${ }^{64}$ den elektronischen Geschäftsverkehr umfassend geregelt hat und zukünftig weitere Voraussetzungen schafft, etwa mit dem geplanten Bürgerportalgesetz. ${ }^{65}$

\section{Leistungsansprüche}

Von der grundgesetzlichen Gewährleistung in Bezug auf die Inhalte des Internets sind verfassungsrechtliche Ansprüche zu unterscheiden, die den Einzelnen in den Stand setzen, auf solche Inhalte zuzugreifen bzw. das Internet zur Verbreitung von Inhalten zu nutzen.

Die Möglichkeit, Inhalte zu verbreiten und einzuholen, war und ist immer durch die intellektuellen und wirtschaftlichen Möglichkeiten der Einzelnen begrenzt. ${ }^{66}$ Das Nutzen von Kommunikationskanälen ist grundsätzlich der Eigeninitiative und dem eigenen Mitteleinsatz überlassen. So garantiert Art. 5 GG bekanntermaßen keine Zuhörerschaft. ${ }^{67}$ Auch hat die Informationsbeschaffung über die (herkömmlichen) Medien seit jeher ihren Preis. Presseerzeugnisse und die Konsumtion des Rundfunks ist nicht kostenlos. Vor diesem herkömmlichen Hintergrund und angesichts der Tatsache, dass Inhalte im Internet (bislang) weitgehend kostenfrei sind, besteht hier keine besondere Problemlage. Auch kann man seine Meinung im Internet viel besser verbreiten, als dies einem Leserbriefschreiber möglich gewesen war und ist. Leistungsansprüche auf der Inhaltsebene, sowohl hinsichtlich der Verbreitung wie auch der Verschaffung, sind deshalb gegenwärtig nicht relevant.

63 Es würde in der Informationsgesellschaft dann nicht nur um ein Recht auf Internet, sondern auch (und vielleicht noch mehr) um ein Recht auf analogen Zugang gehen. Für ein solches sind Parallelen zur Barrierefreiheit für (Seh-)Behinderte zu ziehen oder zu den Regelungen für Analphabeten, wonach (manche) schriftliche Verfahrenshandlungen auch zur Niederschrift erklärt werden können (z.B.

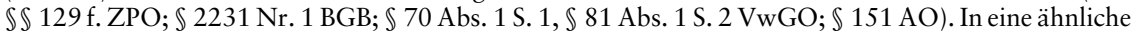
Richtung geht der Hinweis, dass für Schmalbandanschlüsse geeigneten Internetangeboten gewährleistet werden müssten (Mecklenburg, Internetfreiheit (Fn. 2) S. 534).

64 Richtlinie 2000/31/EG v. 8.6.2000 über bestimmte rechtliche Aspekte der Dienste der Informationsgesellschaft, insbesondere des elektronischen Geschäftsverkehrs, im Binnenmarkt (ABl. EG L 178 S. 1-16).

65 BT-Drucks. 16/12598; s. dazu auch P. Hense, in: Goerlich (Fn. 33), S. 73 (87 ff.).

66 So auch E. Schmidt-Jortzig, in: Isensee/Kirchhof (Fn. 51), $\mathbb{1} 162$ Rn. 43 a.E.

67 Zu Recht Greve/Schärdel (Fn. 36) S. 55. 
Gleichwohl ist es nicht ausgeschlossen, Leistungsansprüche auf Internet demokratie-68 oder sozialstaatlich zu begründen. ${ }^{69}$ Denn auch die von staatlicher Hilfe Abhängigen müssen am kulturellen und politischen Leben teilhaben können. Dies wird herkömmlich vor allem durch die Rundfunkgebührenbefreiung ( $\$ 6 \mathrm{RGebStV})$ sichergestellt. Darüber hinaus werden Unterhaltung, Bildung und Kultur nach $\mathbb{S} 2$ Abs. 2 Nr. 8 RSV/S 5 Abs. 1 Abt. 9 u. 10 RBEG sozialrechtlich berücksichtigt. Allerdings beträgt der Satz nur etwa $1,30 €$ pro Tag, reicht also für ein Abonnement einer Tageszeitung nur knapp, für eine Wochenzeitung aber wohl aus. Doch ist hierbei herkömmlicherweise auch etwa die Auslage von Zeitungen in öffentlichen Bibliotheken als Leistungsgewährung zu berücksichtigen. - Auf das Internet übertragen bedeutet dies, dass zukünftig einmal existierender kostenpflichtiger Content bei der Berechnung des Existenzminimums berücksichtigt werden muss, soweit er existenznotwendig ist und nicht kostenlos oder öffentlich-rechtlich erbracht ${ }^{70}$ wird.

\section{Abwehrrechtlicher Schutz}

Gewährleistungs- und Leistungspflichten des Staates in Bezug auf Internetinhalte bestehen also nur eingeschränkt und eher im Wartestand, weil im Rahmen der vorhandenen und als Schmalbandanschluss gewährleisteten Telekommunikationsinfrastruktur jedermann am Internet teilnehmen ${ }^{71}$ kann und die Inhalte weitestgehend kostenlos sind.

Jedoch können diese Möglichkeiten aufgrund von Gesetzen eingeschränkt werden, und sie werden dies auch. Dass das Internet insoweit kein rechtsfreier Raum ist, ist eine Binsenweisheit. ${ }^{72}$ Nicht nur ist das allgemeine Zivil- und Strafrecht im Internet anwendbar, sondern auch die speziellen Regelungen sind Legion. Zu nennen sind neben Spezialvorschriften des Urheber- und Datenschutzrechts das (nicht vollzogene)

68 Eine Rolle kann hier auch die (rechtlich vorausgesetzte) Geschwindigkeit des Informationszugangs spielen. Gegenwärtig haben Wahlentscheidungen (jedenfalls praktisch) einen Tag Karenz, da am Wahltag keine Wahlwerbung mehr gemacht wird (vgl. auch $\ 32$ BWahlG). Der Anspruch eines Betriebsrats auf Internet wird aber auch mit der Notwendigkeit tagesaktueller Information begründet (BAG NJW 2010, S. 1901 (1902)).

69 Siehe bei Starck (Fn. 47), Art. 5 Rn. 19 m.w.N.

70 Vgl. hierzu schon jetzt $\$ 11$ d Abs. 3 RStV i.d.F. d. 12. RÄStV.

71 „Teilnahme“ ist hierbei weit zu verstehen. Davon umfasst ist nicht nur die passive, rezeptive Nutzung (Surfen), sondern auch das Bereitstellen von (eigenen) Inhalten (Homepage). Auch die direkte Kommunikation (Email, auch Internet-Telefonie) fällt hierunter. - Tatsächlich ist die kategorische Trennung von Anbietern und Nutzern, wie sie etwa dem TMG zugrundeliegt, durch das sog. „Web 2.0“, das durch soziale Netzwerke usw. gekennzeichnet ist, überholt (z.B. K.-H. Ladeur, Toward a NetworkOriented Law of the Internet!, GLJ X (2009), S. 1201 (1201 f.)).

72 J. Park, Ehrenschutz im Internet am Beispiel der Hassrede, Hamburg 2006, S. 54, spricht von einem „Klassiker“. 
ZugErschwG ${ }^{73}$ und das (zeitweise) geplante $\mathrm{LöschG}^{74}$ und die bereits existierenden Sperr-Regelungen in den Rundfunk- und Medienstaatsverträgen (s. $\mathbb{\$} 59$ Abs. 3 S. $2 \mathrm{RStV}$ und $\mathbb{} 20$ Abs. $4 \mathrm{JMStV}^{75}$ ). - Da hier die Hauptgefahr für ein inhaltlich akzentuiertes „Recht auf Internet“ liegt, ist inzwischen die Feststellung wichtig, dass das Internet nicht nur kein rechtsfreier, sondern auch kein grundrechtsfreier Raum ist.

\section{Zerklüftetheit der Schutzbereiche}

Der grundrechtliche Schutz der inhaltsbezogenen Nutzung des Internets bemisst sich im Allgemeinen nach Art. 5 und Art. 2 Abs. 1 GG. Selbstverständlich lassen sich aber fast allen Grundrechten Schutzgehalte in Bezug auf Internetsachverhalte entnehmen, so für wirtschaftliche Sachverhalte dem Art. 12 und ggf. Art. 14 GG, hinsichtlich des Missionierens dem Art. 4 GG und dem Art. 17 GG für das Werben um Unterstützer für eine Petition. ${ }^{76}$

Diese Zerklüftung der Grundrechtslandschaft setzt sich innerhalb des Art. 5 Abs. 1 GG, jedenfalls auf der Schutzbereichsebene, fort. ${ }^{77}$ Geschützt ist die Meinungsäußerungsfreiheit, die Meinungsverbreitungsfreiheit und die Informationsfreiheit, und dies jeweils für die Form von Wort, Schrift und Bild (Art. 5 Abs. 1 S. 1 GG). Daneben sind die Pressefreiheit und die Freiheit der „Berichterstattung durch Rundfunk und Film“ besonders geschützt (Art. 5 Abs. 1 S. 2 GG). Diese Gehalte bilden die Struktur des Informationsflusses im Internet freilich nur unvollkommen ab. - Eine allgemeine Kommunikationsfreiheit gibt es grundrechtsdogmatisch nicht, anders als etwa nach Art. 10 Abs. 1 S. 1 EMRK oder nach Art. 11 Abs. 1 GRCh.

\section{a. Meinungsfreiheit}

Entscheidend für die Reichweite des Schutzes des Art. 5 GG ist zunächst und vor allem der Begriff der „Meinung“. Klassische Diskussionsfelder sind die Frage, ob hierunter auch Tatsachenäußerungen fallen, ob falsche und bewusst unwahre Tatsachenäußerungen am Schutz der Meinungsfreiheit teilhaben, ${ }^{78}$ und in welchem

73 Gesetz zur Erschwerung des Zugangs zu kinderpornographischen Inhalten in Kommunikationsnetzen (Zugangserschwerungsgesetz) v. 17.2.2010 (BGBl. I S. 78); dazu Chr. Schnabel, Das Zugangserschwerungsgesetz, JZ 2009, S. $996 \mathrm{ff}$.

74 S. hierzu den bislang nicht offiziell veröffentlichten Entwurf eines „Gesetzes zur Förderung der Löschung kinderpornografischer Inhalte“ (s. die Meldung der Neuen Osnabrücker Zeitung v. 24.4.2010).

75 Auf diese bislang wenig diskutierten Regelungen weist Ladeur, Network-Orientated Law (Fn. 71), S. 1204, hin.

76 S. dazu grundlegend M. Hochhuth, Die Meinungsfreiheit im System des Grundgesetzes, Tübingen 2007, S. $82 \mathrm{ff}$.

77 Diese Zerklüftung positiv als Differenzierung herausstellend allerdings Schulze-Fielitz (Fn. 3), Art. 5 I, II Rn. 40.

78 BVerfGE 99, S. 185 (196); BVerfGE 90, S. 1 (15); BGHZ 139, S. 95 (101); zur a.A. im Schrifttum die Nachw. bei H. D. Jarass, in: ders./B. Pieroth (Hrsg.), Grundgesetz, 10. Aufl., München 2009, Art. 5 Rn. 4; M. Kloepfer, Verfassungsrecht (Fn. 1), Bd. 2, München 2010, $\$ 61$ Rn. 9. 
Umfang Werbung sich auf die Meinungsfreiheit berufen kann. ${ }^{79} \mathrm{Im}$ hier zu behandelnden Internetkontext stellen sich weitere spezifische Fragen, etwa die, ob Programmcode zum Herunterladen ebenfalls als Meinung zu begreifen ist. Problematisch ist auch das für das Internet typische Verlinken und Zitieren fremder Äußerungen, das dem Schutz der Meinungsfreiheit nur unterfällt, wenn es sich nicht um eine falsche Tatsachenäußerung handelt. Betroffen sind hiervon etwa Gerüchte über neue Produkte der Unterhaltungselektronik, die die Grundlage für viele technisch orientierte Online-Portale bilden. Ferner fällt auf, dass die Meinungsäußerungsfreiheit auch die nicht-öffentliche Äußerung schützt, ${ }^{80}$ nicht aber bzw. nur im Reflex den Empfänger einer solchen. ${ }^{81}$

\section{b. Medienfreiheit}

Einem - jedenfalls auf der Schutzbereichsebene - zusätzlichen Schutz dient die besondere Gewährleistung von Presse-, Rundfunk- und Filmfreiheit. Hierdurch werden bestimmt modale Meinungsverbreitungsarten besonders angesprochen. Das Internet ist als ein solcher Modus allerdings nicht erwähnt. ${ }^{82}$

Doch lassen sich einige Meinungsverbreitungsarten im Internet gleichwohl unter die genannten Tatbestandsalternativen subsumieren. Vorherrschend ist dabei eine historische Auslegung der Begriffe: So werden im Ausgangspunkt unter Presse die Erzeugnisse mittels Druckerpresse verstanden, also dinglich verkörperte Papierwerke. Ebenso wird unter „Film“ nur das „Lichtspiel“, im wesentlichen also das Kino, gefasst. Nach diesem Verständnis sind die Internet-Zeitungen und Nachrichten-Portale keine „Presse“ 83 und Video-Portale (Youtube) kein „Film“ im Sinne des Art. 5 Abs. 1 S. 2 GG. Sie können aber dem Begriff des Rundfunks unterfallen, wenn sie sich an die Allgemeinheit richten (1 zu n-Kommunikation). Nicht als Rundfunk eingeordnet werden die sogenannten Telemedien, die zwar für die Allgemeinheit er-

79 Jedenfalls bejaht für Werbung mit (auch) politischem Aussagegehalt, vgl. BVerfGE 71, S. 162 (175); BVerfGE 102, S. 347 (359) - Schockwerbung I; s. auch BVerfGE 107, S. 275 (280); differenzierend P. Lerche, Werbung und Verfassung, München 1967, S. 76 ff.; zusammenfassend K. J. Grigoleit/J. Kersten, Grundrechtlicher Schutz und grundrechtliche Schranken kommerzieller Kommunikation, DVBl. 1996, S. $596 \mathrm{ff}$.

80 M. Kloepfer, Verfassungsrecht (Fn. 78), \$61 Rn. 18.

81 Vgl. BVerfGK 4, S. 305 (309f.); Jarass (Fn. 78), Art. 5 GG, Rn 6; B. Kempen, in: Epping/Chr. Hillgruber (Hrsg.), Grundgesetz, München 2009, Art. 5 Rn. 11.

82 Mecklenburg, Internetfreiheit (Fn. 2) S. 525 f.; Schulze-Fielitz (Fn. 3), Art. 5 I, II, Rn. 200.

83 Kritisch etwa Chr. Möllers, Pressefreiheit im Internet, AfP 2008, S. 241 (243 ff.). 
reichbar sind, dann aber eine Individualkommunikation (1 zu 1) zum Gegenstand haben. ${ }^{84}$

\section{c. Kunst- und Wissenschaftsfreiheit}

Schließlich ist auf die besonderen, inhaltlich zu bestimmenden Schutzbereiche der Kunst- und Wissenschaftsfreiheit hinzuweisen. Hiervon umfasst ist nicht nur die Informationsbeschaffung (Inspirationsquellen für den Künstler, Erkenntnisquelle für den Wissenschaftler), sondern auch der sogenannte „Wirkbereich“, also die Verbreitung von Kunst sowie von wissenschaftlicher Erkenntnis. ${ }^{85}$

\section{d. Nachfrager- und Empfängerschutz teilweise nur nach Art. 2 Abs. 1 GG}

Einen geringeren und dann nicht bei Art. 5 GG zu verortenden Grundrechtschutz erfahren die Nachfrager nach Inhalten, die nicht als „Meinung“ i.S.d. Art. 5 GG qualifiziert werden. Auch fehlt es an einem spezifischen Schutz für Empfänger nichtöffentlicher und unangeforderter Nachrichten und Information. ${ }^{86}$ Auch werden die Nachfrager nach Kunst und wissenschaftlicher Erkenntnis, soweit diese nicht öffentlich zugänglich sind, nicht durch Art. 5 GG geschützt, teilweise aber durch (Wirtschafts-)Grundrechte, jedenfalls aber stets durch die Allgemeine Handlungsfreiheit.

\section{e. Kein Meinungsschutz für Internetdienste}

Auf der Grundrechtsebene fehlt gänzlich ein spezifischer Schutz von Anwendungen (Internetdiensten). Denn Anwendungen sind keine allgemein zugänglichen Meinungen oder auch nur Informationen und deshalb keine Quelle i.S.d. Art. 5 Abs. 1 S. 1 GG. So sind etwa das Homebanking, Versteigerungs-Portale und Online-Spiele

84 Die Einordnung von Internetinhalten als Presse, Rundfunk oder Film ist kein reines Glasperlenspiel. So ist dies in einem föderalen Kontext entscheidend dafür, ob die Regelungskompetenz beim Bund (Telemedien, Art. 74 Abs. 1 Nr. 11 GG [Wirtschaft]; frühere Rahmengesetzgebung für die Presse, Art. 75 Abs. 1 S. 1 Nr. 2 GG a.F.) oder bei den Ländern liegt (Rundfunk). Hiernach bestimmen sich etwa die Möglichkeit von Internetsperren (s. die nur auf Mediendienste anwendbaren $\mathbb{5 9}$ Abs. 3 S. 2 RStV u. $\$ 20$ Abs. 4 JMStV). Auf der Ebene der Grundrechte wird zudem - jedenfalls bislang vom Bundesverfassungsgericht (st. Rspr., s. z.B. BVerfGE 83, S. 238 (295 f.); BVerfGE 87, S. 181 (197)) die Rundfunkfreiheit als eine bloß dienende und deshalb leichter beschränkbare verstanden.

85 Insbesondere mit Blick auf die rechtspolitische Diskussion über Internetsperren von (kinder-)pornographischen Inhalte ist anzumerken, dass solche u.U. der Wissenschafts-, vor allem aber der Kunstfreiheit unterfallen können (BGH, NJW 1990, S. 3026 (3026 f.)). Außer Frage steht freilich, dass der Missbrauch von Kindern weder hinsichtlich des Werk- noch des Wirkbereichs grundrechtlich geschützt ist.

86 Hierbei handelt es sich um die Spam-Konstellation, die bislang vorwiegend unter dem Gesichtspunkt der Abwehr von Belästigungen diskutiert worden ist. Sie enthält aber auch eine Meinungsrelevanz hinsichtlich des Empfängers, der sich dann nicht auf Art. 5 GG berufen kann. Dies kann mit den einstweilen fiktiven Beispiel eines nationalen Spamfilters illustriert werden: Hier würden Nachrichten hoheitlich dem Empfänger vorenthalten werden, die er aber möglicherweise interessant fände...-Dass eine solche Konstellation nicht hypothetisch ist, zeigen die gegenwärtigen Planung des „rechtssicheren elektronischen Briefs“ der DE-Mail, bei der von Viren befallene Nachrichten gekennzeichnet und möglicherweise auch aussortiert werden (vgl. D. Borchers, Elektronische Einschreiben, c't 17/2010, S. $74(74 \mathrm{f.}))$. 
für den privaten Nutzer nur über die allgemeine Handlungsfreiheit geschützt. ${ }^{87}$ In Bezug auf gewaltverherrlichende oder rassistisch konnotierte Spiele können sich hier Wertungsunterschiede zur Freiheit der Verbreitung solcher Inhalte ergeben: Als Spiel verbietbar, als Meinung oder Kunst verfassungshoch geschützt...

\section{f. Unvollkommener Schutzbereich in Bezug auf das Internet}

Der grundrechtliche Schutz des Art. 5 und Art. 2 Abs. 1 GG ist in seiner Zerklüftetheit also unvollkommen und lässt mit Blick auf Internetsachverhalte Lücken und Ungleichgewichte erkennen. ${ }^{88}$ Dieser Befund ist mit Blick auf Schutzgehalte weiterer (Spezial-)Grundrechte noch weiter zu unterstreichen. So sind zum einen nicht alle Inhalte des Internets von dem Schutz des Art. 5 GG erfasst (Programmcode, Internetdienste, Zitate und Links zu unwahren Tatsachenäußerungen). Auch sind - jedenfalls normstrukturell - die Verbreiter von Nachrichten privilegiert, soweit sie als „Rundfunk“ i.S.d. Art. 5 Abs. 1 S. 2 GG bzw. Kunst oder Wissenschaft i.S.d. Art. 5 Abs. 3 S. 1 GG gelten oder soweit sie im nicht-öffentlichen Kontext geäußert werden. Auch ist die unternehmerische und berufliche Nutzung des Internets gegenüber der privaten wegen des Art. 12 Abs. 1 GG stärker abgesichert.

Praktisch wird - das soll nicht unerwähnt bleiben - die Freiheit des Internets auch durch dass Gleichheitsgebot geschützt. Denn ohne einen hinreichenden sachlichen Grund darf hinsichtlich des Zugangs zu Informationen und anderen Inhalten nicht differenziert werden darf; eine solche Differenzierung ist, abgesehen vom Jugendschutz, kaum vorstellbar. Wenn also Inhalte gesperrt werden, dann für die Allgemeinheit, die dann hierüber letztlich politisch zu befinden hat.

87 Für den Diensteanbieter und den unternehmerischen Nutzer tritt daneben noch der Schutz durch Art. 12 GG, ggf. auch der nach Art. 14 GG.

88 Anders Hochbuth, Meinungsfreiheit (Fn. 76), S. 294, der die internetspezifischen Probleme vornehmlich außerhalb des Art. 5 GG sieht. 


\section{Eingriff}

Inhaltliche ${ }^{89}$ Beschränkungen $^{90}$ im Internet - Websperren und Internetfilter ${ }^{91}$ - richten sich meist gegen den Empfänger, der, anders als es dieser Begriff nahelegt, im Internet aber keineswegs eine passive Rolle hat, sondern aktiv auf Inhalte zugreift. Es gibt aber auch Eingriffe in die Verbreitungsfreiheit. Hier ist an die gegenwärtig politisch favorisierte Löschverfügung für Kinderpornographie zu denken ${ }^{92}$ und die medienrechtlichen Sperrverfügungen ( $\mathbb{S} 59$ Abs. 2-4 RStV; $\mathbb{2} 20$ Abs. 4 JMStV). Ein aktuelles Beispiel waren auch die (faktischen) Bemühungen der u.s.-amerikanischen Regierung Ende 2010, die Internetplattform Wikileaks von der Öffentlichkeit und von ihrer (finanziellen) Unterstützung zu trennen.

\section{Schranke}

Solche Eingriffe in die Meinungsfreiheit dürfen aufgrund allgemeiner, nicht gegen eine bestimmte Meinung gerichteter Gesetze, zum Schutze der Jugend und zum Schutze der Ehre erfolgen. Die Wissenschafts- und Kunstfreiheit ist (textlich) unbeschränkt, was eine verfassungsunmittelbare Beschränkbarkeit freilich nicht ausschließt. Die Allgemeine Handlungsfreiheit unterliegt (schon) der Schranke der (verfassungsmäßigen) Rechtsordnung. ${ }^{93}$

89 Das verkennt nicht und schließt nicht aus, dass Sperr- und Filtermaßnahmen oft auf der technischen Ebene ansetzen (aktuelle Übersicht etwa bei A. Koreng, Zensur im Internet, Baden-Baden 2010, S. 134 ff. - J. Zittrain/J. G. Palfrey unterscheiden (mindestens) fünf Arten rechtlicher Kontrolle des Internets: Regulierung von Inhalten, Genehmigungserfordernisse für Diensterbringer und TK-Dienstleister, Haftungsregeln zulasten von ISPs bei Rechtsverstößen von Nutzern, Registrierungserfordernisse für Internetnutzer, (tatsächliche oder auch nur scheinbare) Internetüberwachung, die ebenfalls zur einer Verhaltensänderung der Nutzer führt (J. Zittrain/J. G. Palfrey, Internet Filtering: The Politics and Mechanisms of Control, in: R. J. Deibert/J. G. Palfrey/R. Rohozinski/J. Zittrain (Hrsg.), Access Denied, Cambridge, Mass; MIT Press, 2008, S. 29 (32 f.)).

90 Beschränkungen der allgemeinen Zugänglichkeit sind keine Frage des Schutzbereichs, sondern immer ein Eingriff (zuerst $P$. Lerche, Informationsfreiheit, in: H. Kunst/S. Grundmann/W Schneemelcher/R. Herzog (Hrsg.), Evangelisches Staatslexikon, Stuttgart, 1966, Sp. 785 (786); ihm folgend BVerfGE 27, S. 71 (83 f.) - Leipziger Volkszeitung; weitere Nachw. bei Starck (Fn. 47), Art. 5 GG, Rn. 44 Fn. 142).

91 Solche Kommunikationsbarrieren sind keine neues Phänomen und kein Spezifikum des Internets. Vielmehr finden sie in der (deutschen) Geschichte ihre Entsprechung in den Störsendern gegen die BBC im II. Weltkrieg und gegen den RIAS in der Zeit des Kalten Krieges. Und auch die westdeutsche Geschichte ist insoweit nicht frei von dunklen Flecken. Genannt werden kann hier das (staatsschutzrechtlich motivierte und gegen östliche Propaganda gerichtete) Überwachungsgesetz v. 1961 („Gesetz zur Überwachung strafrechtlicher und anderer Verbringungsverbote“ (GÜV) v. 24.5.1961 (BGBl. I S. 607); dazu auch BVerfGE 71, S. 27 ff. - Leipziger Volkszeitung). - In der jüngeren Diskussion werden Internet-Sperren (abgeschwächt) als Warnhinweise (Leyens Stoppschild, Warnhinweis für Urheberrechtsverletzer) und Kennzeichnungspflichten (etwa hinsichtlich der Jugendgeeignetheit von Online-Inhalten nach $\$ 5$ Abs. $2 \mathrm{JMStV}$ i.d.F. d. 14. RÄStV) diskutiert.

92 Pornographie wird verbreitet als Grund für die Einführung von Internet-Überwachungssystemen angegeben, die hierfür geschaffene Infrastruktur kann dann aber auch für andere Zwecke eingesetzt werden (hierzu R. Faris/N. Villeneuve, Measuring Global Internet Filtering, in: Deibert/Palfrey/Rohozinski/Zittrain, Access Denied (Fn. 89), S. 5 (24 f.), speziell zu der Lage in Vietnam).

93 Anders als die völkerrechtlichen Menschenrechtskodifikationen niedergelegt ist, unterliegt die Meinungsäußerungsfreiheit keinen besonderen Pflichten (Schranken) und ist auch nicht Gegenstand besonderer Verantwortung (vgl. Art. 19 Abs. 3 IPbpR). Auch so etwas wie einen allgemeinen Missbrauchsvorbehalt kennt das Grundgesetz, wie sich im Gegenschluss aus Art. 18 GG ergibt, nicht. 
Die Schranke der allgemeinen Gesetze ist hauptsächlich in Bezug auf politische Inhalte relevant. Hier ging es in der Vergangenheit vor allem um nationalsozialistische Hetze. ${ }^{94}$ Hinsichtlich des Jugendschutzes, um dessen willen Kinderpornographie bekämpft wird, muss in dogmatischer Hinsicht angemerkt werden, dass sich diese Schrankenbestimmung nur auf Jugendliche als Empfänger, nicht auch auf Kinder als Opfer bezieht. ${ }^{95}$ Hinsichtlich des Ehrschutzes kommt es auf den jeweiligen Einzelfall an.

Werden solche Inhalte allerdings als wissenschaftliche Äußerung oder als Kunst verbreitet, können Beschränkungen nur aus der Verfassung selbst hergeleitet werden (verfassungsimmanente Schranken). Insbesondere Manga-Comics (gezeichnete Kinderpornographie) und die - hier jetzt um des Beispiels willen denkbare - wissenschaftlich fundierte Verneinung des Holocaust markieren die Grenze der Einschränkbarkeit. Gerade die Kategorien von Inhalten, über deren Sperrung man am ehesten politischen Konsens herstellen könnte (Nazi-Propaganda, Kinderpornographie), sind hinsichtlich der Schrankenbestimmungen der Meinungsfreiheit alles andere als unproblematisch.

\section{Zensurverbot}

Speziellen Schranken-Schranken, wie sie insbesondere hinsichtlich des Art. 5 GG in Gestalt des Zensurverbots bestehen, laufen in Bezug auf das Internet weitgehend leer. Denn das Zensurverbot (Art. 5 Abs. 1 S. 3 GG), das herkömmlich als Verbot der Vorzensur verstanden wird, ist im Internet, in dem die Verbreiter von Information schon wegen ihrer großen Zahl und der räumlichen Beweglichkeit nicht zu fassen sind, kaum je relevant. ${ }^{96}$ Internetsperren und Filter sind klassische Fälle der „Nachzensur“, ebenso die nachträgliche Löschung. - Auch schützt das Verbot der Vorzensur wieder nur die Anbieter von Information (Presse, Rundfunk, Film), nicht auch den Nachfrager. ${ }^{97}$

\section{Internetsperre als Grundrechtsverwirkung?}

$\mathrm{Zu}$ den noch wenig thematisierten Aspekten der Meinungsfreiheit, die sich im $\mathrm{Zu}$ sammenhang mit „Internetsperren“ besonders stellen, gehört die Frage, ob es sich hierbei um eines Frage der Grundrechtsverwirkung handelt. Denn ein Regelung wie

94 Dazu nun BVerfGE 124, S. 300 ff.; zum Diskussionsstand bis dahin s. U. Battis/K. J. Grigoleit, Rechtsextremistische Demonstrationen und öffentliche Ordnung - Roma locuta?, NVwZ 2001, S. $121 \mathrm{ff}$.

95 Vgl. etwa bei Starck (Fn. 47), Art. 5 GG, Rn. 204-208, 343-345, anders aber wohl a.a.O., Rn. 253. - Internetfilter aus Jugendschutzgründen sind also nur insoweit erlaubt, als sie auf das Alter der Empfänger abstellen, nicht auf das Alter der Dargestellten. - Möglicherweise kann aber eine Beschränkung der Meinungsfreiheit - im Internet, aber auch in der Offline-Welt - in Sachen Kinderpornographie auf den Schutz der Ehre der missbrauchten Kinder gestützt werden oder auf die Schutzpflicht aus Art. 6 Abs. 2 GG.

96 Gucht, Das Zensurverbot im Gefüge der grundrechtlichen Eingriffskautelen, Frankfurt 2000, S. 133 f.

97 BVerfGE 27, S. 88 (102) - Der Demokrat. 
etwa nach der französischen loi Hadopi98, nach der der Zugang zum Internet für eine bestimmte Zeit zu gesperrt werden kann, ist nichts anderes als die Auferlegung einer „nach Art und Dauer genau bezeichnete[n] Beschränkung[...]“ ( $\mathbb{\$} 39$ Abs. 1 S. 2 BVerfGG). Nach dem hier zugrunde gelegten Schichtenmodell handelt es sich bei dem Sperren des technischen Zugangs zunächst einmal um eine bloße Beschränkung der Allgemeinen Handlungsfreiheit (Art. 2 Abs. 1 GG). Wenn und sobald diese allerdings entstehenssichernd für die Meinungsfreiheit ${ }^{99}$ wäre $^{100}$ - etwa, weil das Internet als Verbreitungskanal essentiell ist -, läge hierin ein Verstoß gegen die Schranken-Schranke des Art. 18 GG, der einen solchen Ausspruch ausschließlich dem Bundesverfassungsgericht zuweist.

\section{Strukturelle Schwächen des grundrechtlichen Schutzes des Internets}

Bei der Analyse der Regelungen, die den Schutz eines verfassungsrechtlichen „Rechts auf Internet“ zum Gegenstand haben, fallen nicht nur die eben dargestellten Lücken auf, sondern auch strukturelle Schwächen.

So ist der Schutz der Verbreiter von Inhalten im nicht-öffentlichen Kontext, durch die Medien („Rundfunk“) und von Kunst und Wissenschaft stärker ausgeprägt als die der Nutzer und Nachfrager, jedenfalls auf der Schutzbereichsebene (asymmetrischer Schutz zugunsten von Inhaltsanbietern). Hier spiegelt sich weder die Interaktivität des Internets, ${ }^{101}$ insbesondere in Sozialen Netzen (Web 2.0), noch die im Vergleich zur Offline-Welt viel aktivere Rolle der Nutzer wieder. Die Schutzkonzeption ist noch ganz dem Kommunikationsmodell des 20. Jahrhunderts verhaftet.

Ferner zeigt sich in diesem Zusammenhang eine normstrukturelle Schwäche des auf den Einzelnen ausgerichteten Grundrechtschutzes. Es wird nämlich immer nur der jeweils von einer Maßnahme Betroffene bei der Grundrechtsprüfung betrachtet. Doch beruht das Internet - wie jedes andere Netzwerk auch - auf der Verbindung und Verknüpfung aller Akteure miteinander. Jeder, der in seiner Meinungsäußerungs- oder in seiner Informationsfreiheit beschränkt ist, vermindert für alle anderen den Wert des Internets als Informationsquelle oder Plenum.

\section{Struktur eines Rechts auf Internet}

Ein Recht oder gar ein Grundrecht auf Internet gibt es in dieser ausdrücklichen Form nicht. Es handelt sich dabei vielmehr um einen rechtspolitischen oder auch rechtswissenschaftlichen Sammelbegriff für grundrechtliche Gehalte in Bezug auf eine mo-

98 S. oben Fn. 36.

99 Gleiches gilt für andere in Art. 18 GG genannte Grundrechte.

100 Zur strukturell vergleichbaren Situation, dass landesrechtliche Vorschriften eine dem Aberkennen eines Grundrechts gleichkommende Regelungen enthalten, BVerfGE 10, S. 118 (123 f.).

101 Früh schon Mecklenburg, Internetfreiheit (Fn. 2), S. 529 („Dialogorientierung“). 
derne (Kultur-)Technik. Das bedeutet allerdings nicht, dass das „Recht auf Internet“ keine dogmatischen Konturen hätte.

\section{Unterschied zwischen technischem und inhaltlichem „Recht auf Internet“}

Die hier zugrunde gelegte Unterscheidung zwischen technischer und inhaltlicher Ebene hat deutlich werden lassen, dass beim „Recht auf Internet“ auf der technischen Ebene Gewährleistungs- und Leistungsaspekte überwiegen. Grundrechtsbeschränkungen sind hier selten, der Grundrechtsschutz ist schwach. - Auf der inhaltlichen Ebene sind dagegen, schon wegen der Freiheit der Meinungskampfs und des Gebots der Staatsferne, Gewährleistungs- und Leistungsgehalte von geringerer Bedeutung. Eingriffe auf der Inhaltsebene haben eine große Relevanz, was sich auch in dem erhöhten Grundrechtschutz jedenfalls in Bezug auf „Meinungen“ und die klassischen „Medien“ zeigt.

\section{Grundsatz des freien Informationsflusses}

Die weitgehenden objektiven Gewährleistungen auf der technischen Ebene der Telekommunikation bedeuten zunächst einen objektiven Grundsatz der Internetfreiheit und des freien Informationsflusses. Beschränkungen des Internets sind deshalb immer die begründungsbedürftige Ausnahme. Diese Aussage hat über den Bereich der individuellen Grundrechtsbeschränkung hinaus Bedeutung. Sie bedeutet etwa für den (deutschen) Staat, dass er sich grundsätzlich Handlungen enthalten muss, die das Internet beschränken. Hier ist vor allem an internationale Verhandlungen und Vereinbarungen zu denken, wie sie zuletzt etwa im Zusammenhang mit dem ACTAAbkommen diskutiert worden sind, ${ }^{102}$ oder auch eine Kooperation der Bundesrepublik Deutschland mit der U.S.-Regierung bei der Isolierung der Internetplattform Wikileaks im Nachgang zur sogenannten „Cablegate“ Ende 2010.

\section{E. Normative Umsetzung eines Rechts auf Internet}

Es zeigt sich: Das Grundgesetz ist an das Internet noch nicht angeschlossen. Es ist vielmehr teilweise noch der Welt des Fernmeldens (Art. 10 Abs. 1 GG: „Fernmeldegeheimnis“) verhaftet. ${ }^{103}$ Das kann bei seinem Alter von über sechzig Jahren nicht überraschen. Doch auch Sechzigjährige können sich technischen Neuerungen anpassen. Hierfür gibt es mehrere Wege: Möglich ist ein Aufbautraining (juristisch: Interpretation) oder eine Operation (juristisch: Verfassungsänderung). Schließlich ist

102 S. zur Haltung der Bundesregierung BT-Drucks. 17/1322 v. 8.4.2010; vgl. auch die Entschließung des Bundesrats (BR-Drucks. 201/10 v. 14.4.2010) und die des Europäischen Parlaments v. 24.11.2010.

103 Es soll nicht verkannt werden, dass das Grundgesetz an anderen Stellen (Art. 73 Abs. 1 Nr. 7 , Art. $87 \mathrm{f} \mathrm{GG)} \mathrm{zumindest} \mathrm{in} \mathrm{der} \mathrm{Welt} \mathrm{der} \mathrm{Telekommunikation} \mathrm{bereits} \mathrm{angekommen} \mathrm{ist.} \mathrm{Jedenfalls} \mathrm{ist}$ dieser begriffliche Nachlauf für Verfassungen typisch: So kannte etwa die WRV zwar schon „Lichtspiele“ (Art. 118 Abs. 2 WRV), noch nicht aber den Rundfunk (dann erst Art. 5 Abs. 1 S. 2 GG). 
auch an die pragmatische Lösung der Brille (juristisch: einfaches Gesetz) zu denken. ${ }^{104}$

Einer Verfassungsänderung, also der textlichen Schaffung eines „Grundrechts auf Internet“, steht - außer dem Erfordernis verfassungsändernder Mehrheiten - nichts im Wege. Allerdings müsste man sich zunächst auf den Inhalt eines solchen Internetgrundrechts verständigen. Textlich könnte man sich hierfür an Art. 10 EMRK und Art. 11 GRCh orientieren, doch darf dann der Schutz des telekommunikativen Zugangs nicht übersehen werden. - Vorzugswürdig wäre es, wenn sich die Unterscheidung und das Zusammenspiel von technischem Zugang und Inhalt dann auch auf der Verfassungsebene wiederfinden würden. ${ }^{105}$

Denkbar und pragmatisch naheliegend wäre daneben eine einfachgesetzliche Regelung, also eine gesetzliche Sehhilfe, die die verfassungsrechtliche Alterskurzsichtigkeit des Grundgesetzes korrigiert. Solche einfachgesetzlichen Regelungen hätten allerdings keinen Verfassungsrang. Doch könnten sie durch Einbettung in strukturell änderungsfestere Kodifikationen „gehärtet“ werden, wie dies etwa von Michael Kloepfer in Form eines Informationsgesetzbuchs (IGB) vorgeschlagen ${ }^{106}$ und von der ehemaligen Bundesjustizministerin Brigitte Zypries in Form eines Internetgesetzbuch (NetGB) vor kurzem wieder ins Gespräch gebracht worden ist. ${ }^{107}$

Dem „Recht auf Internet“ ist mittelfristig am meisten gedient, wenn man die Interpretationsspielräume des Grundgesetzes nutzt. ${ }^{108}$ Bei deren Ausfüllung können die herausgearbeiteten objektiven Gehalte der Internetfreiheit nutzbar gemacht werden. In diesem Rahmen könnte etwa das Tatbestandsmerkmal „Quelle“, zu der jedermann ungehindert zum Zwecke der Unterrichtung Zugang hat (Art. 5 Abs. 1 S. 1 GG), über den Meinungs- und Informationskontext hinaus auf alle Inhalte des Internets einschließlich der Dienste und Anwendungen erweitert werden. ${ }^{109}$ „Presse“, „Rundfunk“ und Film“ könnten umfassend als allgemeine „Medienfreiheit“ verstanden werden ${ }^{110}$ oder um eine „Internetfreiheit“ ergänzt werden. ${ }^{111}$ Auch

104 Ähnliche Metaphern verwendet S. Baer, Braucht das Internet ein Update?, auf: Netzpolitischer Kongress der GRÜNEN, 13.11.2010 (www.youtube.com/watch?v=mcfHCv2LOPo): „Update“, „Training".

105 In diesem Zusammenhang sei auch darauf hingewiesen, dass die Schaffung neuer Grundrechtsbestimmungen gleichzeitig auch neue Grundrechtsschrankenbestimmungen bedeutet. Das Niveau des Grundrechtschutzes wird also durch ein neues Grundrecht nicht unbedingt angehoben.

106 Hierzu etwa M. Kloepfer, Informationsrecht, München 2002, $\mathbb{1}$ Rn. 93.

107 F.A.Z. v. 7.4.2010, S. 4; s. auch den Vorschlag eines Codex Digitalis (Sommerakademie des ULD 2010, https://www.datenschutzzentrum.de/sommerakademie/2010).

108 Zur Leistungsfähigkeit der Grundrechtsinterpretation, insb. im Hinblick auf Art. 5 GG, Schulze-Fielitz (Fn. 3), Art. 5 I, II, Rn. 7.

109 So etwa Köppen, Grundrecht (Rn. 41), S. 107.

110 A. Koreng, Zensur (Rn. 89), S. 96ff.

111 Mecklenburg, Internetfreiheit (Fn. 2), S. $525 \mathrm{ff}$. 
könnte man unter Zugrundelegung des Gedankens des „Grundrechtsnetzes“112 Beschränkungen der strukturell weniger geschützten Empfänger als Beschränkung aller Beteiligten verstehen. ${ }^{113}$ Auch die Beschränkung des Zensurverbots auf die Vorzensur könnte überdacht werden. ${ }^{114}$ - Die meisten dieser Interpretationen finden sich, mehr oder weniger prominent vertreten, schon heute im Schrifttum und teilweise auch in der Rechtsprechung des Bundesverfassungsgerichts.

Methodisch anders und auch etwas kühner als die erweiternde Interpretation einzelner Tatbestandsmerkmale wäre die Übertragung der Schranken des Art. 5 Abs. 2 GG in toto auf die nur von Art. 2 Abs. 1 GG geschützten informationsbezogenen Freiheitsbetätigungen (Empfang von Individualinformation im Internet, $\mathrm{Zu}$ gang zu Kunst, Nutzen von Anwendungen).

Doch bestehen Probleme des „Grundrechts auf Internet“ nicht nur hinsichtlich des Schutzes, sondern auch hinsichtlich der unzureichenden Schranken. Hier wäre dann durchaus mit einer methodischen Großzügigkeit - zu überlegen, sich hinsichtlich eines Rechts auf Internet von der verfassungsrechtlichen Garantie der Freizügigkeit in Art. 11 GG inspirieren zu lassen. ${ }^{115}$ Obwohl die Schrankenbestimmungen der Freizügigkeit deutlich von den Problemen der unmittelbaren Nachkriegszeit ${ }^{116}$ geprägt sind, passen sie überraschend gut auf Internetsachverhalte, teilweise auch besser als die des Art. 5 GG. So würde über den „Schutz der freiheitlichen demokratischen Grundordnung“ extremistische Propaganda abgedeckt werden. Die Beschränkbarkeit bei „,besonderen Lasten der Allgemeinheit“ würde eine Fair Use-Policy etwa hinsichtlich der Bandbreitennutzung ermöglichen. Der „Schutz der Jugend vor Verwahrlosung“ würde besser als der bloße Jugendschutz des Art. 5 Abs. 2 GG die Bekämpfung der Kinderpornographie erfassen. Und die „Seuchengefahr“ lässt wenngleich nur im übertragenen Sinne - die Bekämpfung von Computerviren als Schrankenbestimmung möglich erscheinen. - Auch strukturell ist die Freizügigkeit des Art. 11 GG hinsichtlich des grundrechtlichen Schutzes des Internets besser ge-

112 Ausführlich G. Kutzschbach, Grundrechtsnetze, Frankfurt 2004, insb. S. 154 f. - Hieran knüpfen jedenfalls begrifflich die „Grundrechtsermöglichungsnetze“ (M. Kloepfer, Vielfaltsicherung durch Ebenentrennung in der Massenkommunikation, Baden-Baden 2010, S. 30, 50) und die „Grundrechtsverwirklichungsnetze“ (M. Kloepfer, Presse-Grosso unter dem Schutz von Verfassungsrecht und Europarecht, Baden-Baden 2000, S. 57-59) an.

113 Zum Postulat der Schrankenidentität von Grundrechtsausübung und der hierfür vorausgesetzten (allerdings eigenen) Handlungen M. Kloepfer (Fn. 43), S. 31.

114 Hierzu jüngst eingehend A. Koreng, Zensur (Rn. 89), S. $212 \mathrm{ff}$.

115 Vgl. zu einer ähnlichen, von ihm als „Analogie“ bezeichneten Übertragung des räumlichen Art. 13 GG auf den virtuellen Sachverhalt der Onlinedurchsuchung J. Rux, Ausforschung privater Rechner durch die Polizei- und Sicherheitsbehörden, JZ 2007, S. 285 (293 f.).

116 Die Schrankenbestimmung des Schutzes der freiheitlichen demokratischen Grundordnung ist allerdings erst im Zusammenhang mit den Notstandsgesetzen (G. v. 24.6.1968, BGBl. I S. 709) eingefügt worden (dazu Kloepfer, Verfassungsrecht (Fn. 1), $\$ 28$ Rn. 85). 
eignet als Art. 5 GG, weil sie auf den individuell sich Bewegenden, ${ }^{117}$ nicht auf den statischen Anbieter abstellt. - Allerdings darf bei der Wiederentdeckung des Art. 11 GG als „Netzgrundrecht“ nicht übersehen werden, dass es sich zum einen um ein bloßes Deutschengrundrecht handelt, und zum anderen auf das Bundesgebiet beschränkt wäre, was dem weltumspannenden Internet nicht gerecht wird.

\section{F. Fazit}

Gibt es also ein „Recht auf Internet“? In dieser einfachen und plakativen Form nicht. Die rechtliche Analyse hat gezeigt, dass die unterschiedlichen Ebenen und Aspekte des Internets verfassungsrechtlich unterschiedlichen Regelungen unterliegen, die dann auch ein unterschiedliches Schutzniveau haben. Dabei fehlt es vor allem an einem akzentuierten Schutz der telekommunikativen Netznutzungsfreiheit. Und der inhaltsbezogene Grundrechtschutz ist strukturell auf die massenmedialen Anbieter sowie Künstler und Wissenschaftler zugeschnitten. Das Internet als „Massenmedium “ im eigentlichen Sinne - als Medium der Massen - spiegelt sich im Grundgesetz nicht wider. Insoweit sind dessen Regelungen nicht mehr auf der Höhe der Zeit, und auch noch nicht alle Verfassungsinterpreten.

Doch ist dies kein Grund für verfassungsändernden Aktionismus. Durch die bereits bestehende objektivrechtliche Gewährleistung der telekommunikativen Infrastruktur sind jedenfalls die Grundlagen eines „Grundrechts auf Internet“ gesichert. Problemfelder bestehen vornehmlich in Bezug auf die inhaltliche Nutzung, sowohl hinsichtlich der Enge des Schutzbereichs wie auch in Bezug auf die nicht alle heutigen Erscheinungen berücksichtigenden Schrankenbestimmungen. Beides hat aber kein solches Ausmaß, dass zu einer verfassungstextlichen „Notoperation“ geschritten werden müsste. Und eine bloße „Schönheitsoperation“, das bloße textliche Einfügen eines „Rechts auf Internet“, ist (politische) Geschmacksfrage. Es spricht viel dafür, dass die „alte Dame Grundgesetz“ in ihrer gegenwärtigen Verfassung rüstig genug ist, um mit ihr ins Netz zu gehen.

117 S. a. den bekannten Aufsatz von M. Ronellenfitsch, Mobilität: Vom Grundbedürfnis zum Grundrecht?, DAR 1992, S. 321 ff., und D. Kettler, Grundrecht auf Mobilität, Berlin 2005. 